Conclusions In our study, the majority of children was not hospitalised, which is in agreement with the recent epidemiologic trends in Clostridium difficile infection. Antibiotic exposure remains the most common and modifiable risk factor, emphasising the importance of searching CD in this group of children.

\section{PO-0221 EARLY DIAGNOSIS OF SEVERE ISRAELI SPOTTED FEVER}

${ }^{1}$ S Bota, ${ }^{2} \mathrm{R}$ De Sousa, ${ }^{3} \mathrm{~L}$ Ventura, ${ }^{1} \mathrm{C}$ Gouveia. ${ }^{1}$ Pediatric Infecciology Unit, Hospital Dona Estefânia CHLC, Lisboa, Portugal; ${ }^{2}$ Center for Vectors and Infectious Diseases Research, National Institute of Health Dr Ricardo Jorge, Águas de Moura, Portugal; ${ }^{3}$ Pediatric Intensive Care Unit, Hospital Dona Estefânia CHLC, Lisboa, Portugal

10.1136/archdischild-2014-307384.874

Introduction Israeli spotted fever (ISF) is caused by Rickettsia conorii Israeli spotted fever strain. In Portugal, it was first described in 1999.

Case report A twelve year old adolescent girl was admitted during summer with fever, macular rash (including palms and plants), mild headache, vomits and intense myalgia for three days. She had daily contact with dogs and lived in a rural area in the south of Portugal, but had no history of tick bites or eschar. Within $12 \mathrm{~h}$ she was in septic shock with multiorgan dysfunction (hypotension, obnubilation, leukopenia, thrombocytopenia, coagulopathy, respiratory distress, acute renal failure, hepatic dysfunction, hyperbilirubinemia and polyserositis) and was transferred to the intensive unit care. Empirical treatment with doxycicline, ceftriaxone, flucloxacillin and clyndamicyn was initiated. Rickettsial infection was confirmed by serology (over fourfold title increase by indirect immunofluorescence, four weeks after the acute illness - IgM >1024; IgG >4096) and by PCR. Sequencing confirmed the infection caused by $R$. conorii Israeli spotted fever strain. The adolescent evolved favourably with no sequelae.

Discussion Severe cases of Israeli spotted fever have been increasingly reported, mostly in adults. In children, it is usually a mild disease. The mechanism by which ISF strain causes more severe illness remains to be determined. The patient's epidemiology and typical rash facilitated the early clinical diagnosis and prompt empirical treatment, which was probably crucial. The absence of an inoculation eschar should not delay the diagnosis.

\section{PO-0222 THE PATHOGENIC EFFECTS OF GARDNERELLA VAGINALIS ON THE A549 HUMAN ALVEOLAR EPITHELIAL CELL LINE}

${ }^{1} \mathrm{~F}$ Cheah, ${ }^{2} \mathrm{KK}$ Wong, ${ }^{1} \mathrm{FL}$ Wong, ${ }^{2} \mathrm{H}$ Salasawati. ${ }^{1}$ Paediatrics, Universiti Kebangsaan Malaysia Medical Center, Kuala Lumpur, Malaysia; ${ }^{2}$ Medical Microbiology and Immunology, Universiti Kebangsaan Malaysia Medical Center, Kuala Lumpur, Malaysia

\subsection{6/archdischild-2014-307384.875}

Background Gardnerella vaginalis is one of the commonest organisms that causes bacterial vaginosis, which is also implicated as a risk factor of preterm birth. This bacterium is also considered the second commonest cause of intrauterine inflammation (chorioamnionitis). Although it creates the characteristic "clue cells" when infecting the vaginal epithelium, the evidence of its pathogenicity on other epithelial surfaces is lacking.

Aim To study the pathogenic changes on the human respiratory tract epithelium as the basis for the understanding of Gardnerella vaginalis-induced fetal lung inflammation, which may occur in preterm intrauterine infection.
Methods A549 ${ }^{\circledR}$ ATCC Human alveolar basal epithelial cell line was grown over an average period of 48-72 $\mathrm{h}$ before exposure to Gardnerella vaginalis serotype ATCC ${ }^{\circledR} 14018^{\mathrm{TM}}$. The multiplicity of infection (MOI) of 100 was used to infect the cell line over a period of $4 \mathrm{~h}$. Adherence, apoptosis and cytotoxicity changes were studied using immunofluorescence and light microscopy. Comparisons were also made to E.coli and GBS, the common pathogens causing neonatal sepsis.

Results Gardnerella vaginalis showed similar adherence to E. coli. It has moderate cytotoxicity when compared to GBS. At 4h, co-culturing Gardnerella vaginalis with A549 cell line consistently exhibited the presence of apoptosis in more than $50 \%$ of the cells as shown using the TUNEL assay. Cytotoxicity was confirmed morphologically with cellular features of pyknosis and elevated LDH in culture supernatant.

Conclusions Gardnerella vaginalis exerts some characteristic changes of infection on respiratory epithelium with signs of cytotoxicity, suggesting that the fetal lung could be similarly affected when this bacterium causes intrauterine infection.

\section{PO-0224 ROTAVIRUS GASTROENTERITIS AND NOSOCOMIAL ROTAVIRUS GASTROENTERITIS AMONG CHILDREN AGED UNDER 5 YEARS IN UNITED ARAB EMIRATES: EPIDEMIOLOGY, CLINICAL PROFILE, DEMOGRAPHIC CHARACTERISTICS AND SEVERITY}

${ }^{1} \mathrm{~J}$ Cheriathu, ${ }^{2} \mathrm{~L}$ Jenny John, ${ }^{1} \mathrm{E}$ Ignatius Dsouza, ${ }^{1} \mathrm{M}$ Shamseldeen, ${ }^{2} \mathrm{~A}$ Mathur. ${ }^{1}$ Pediatrics, Gulf Medical University, Ajman, United Arab Emirates; ${ }^{2}$ Pharmacology, Gulf Medical University, Ajman, United Arab Emirates

\subsection{6/archdischild-2014-307384.876}

Introduction Rotavirus is a leading worldwide cause of acute gastroenteritis (AGE) in young children. This study done to estimate the burden of overall acute gastroenteritis, Rotavirus gastroenteritis (RVGE) and nosocomial RVGE in hospitalised children younger than 5 years of age, and to assess the age and seasonal distribution; duration of hospitalisation and additional hospitalisation associated with hospital-acquired RVGE.

Material and methods A cross-sectional, hospital based study was carried out among hospitalised children with acute gastroenteritis of age $<5$ years between 2011 and 2012. Demographic profile, clinical characteristics, prior hospitalisation were analysed using SPSS version 20 software. Chi-square test and t-test were used to compare variables.

Results Total Paediatric Admissions ( $<5$ yrs excluding newborn): 2783

RVGE as well as Nosocomial RVGE peaks were observed in the months of January, February and April. The other AGE was noted to peak around May and November months. Nosocomial RVGE increases the duration of hospital stay 6 days vs 2 days. Conclusion RVGE is highly contagious, and the efficiency of existing prevention measures (such as handwashing, isolation and cohorting) is variable because of numerous barriers to implementation. Prevention of RV infection by mass vaccination could

Abstract P0-0224 Table 1

\begin{tabular}{llll}
\hline & AGE & RVGE & Nosocomial RVGE \\
\hline Total number & 970 & 240 & 27 \\
Male: Female (\%) & $56: 44$ & $55: 45$ & $68: 32$ \\
Mean age & $23.9(15.2)$ & $23.2(14.6)$ & $18(12)$ \\
Nationality (most common) & EGYPT (18\%) & EGYPT (22\%) & EGYPT (28\%) \\
\hline
\end{tabular}

